\section{International Scientific Journal Theoretical \& Applied Science}

p-ISSN: 2308-4944 (print) e-ISSN: 2409-0085 (online)

Year: 2015 Issue: 04 Volume: 24

Published: $30.04 .2015 \quad$ http://T-Science.org

SECTION 31. Economic research, finance, innovation, risk management.
Elena Evgenevna Dosuzheva

Senior Lecturer,

Department of Economic Computer Science,

Novosibirsk State Technical University,

Russia

dosuzheva@gmail.com

Yurij Vasilievich Kirillov

Candidate of technical sciences, associate professor

Department of Economic Computer Science,

Novosibirsk State Technical University,

Russia

$\underline{\text { kirillov_yu@ngs.ru }}$

\title{
ON THE QUESTION OF TAKING INTO ACCOUNT INFLATION WHEN ASSESSING OF INVESTMENT PROJECTS EFFICIENCY
}

\begin{abstract}
In the article the question of taking into account the inflation component in the process of evaluation of the investment project is considered. In the paper the diagram of the sensitivity analysis of the investment project in two versions: with inflation, excluding inflation are shown. The impact of inflation on net present value of an investment project is analysed.

Key words: Investment, efficiency, innovation, efficiency evaluation, inflation, management, decision-making, investment project.

Language: Russian

Citation: Dosuzheva EE, Kirillov YV (2015) ON THE QUESTION OF TAKING INTO ACCOUNT INFLATION WHEN ASSESSING OF INVESTMENT PROJECTS EFFICIENCY. ISJ Theoretical \& Applied Science 04 (24): 236-241.

Soi: http://s-o-i.org/1.1/TAS*04(24)43 Doi: crossef http://dx.doi.org/10.15863/TAS.2015.04.24.43

\section{К ВОПРОСУ ОБ УЧЕТЕ ИНФЛЯЦИИ ПРИ ОЦЕНКЕ ЭФФЕКТИВНОСТИ ИНВЕСТИЦИОННЫХ ПРОЕКТОВ}

Аннотация: В статье рассмотрен вопрос учета инфляциионной составляющей в процессе оценивания эффективности инвестиционного проекта. В работе приведена схема анализа чувствительности инвестиционного проекта в двух вариантах: с учетом инфляции и без учета инфляции. Проанализировано влияние инфляции на показатель чистого приведенного дохода инвестиционного проекта.

Ключевые слова: Инвестиции, эффективность, инновации, оченка эффективности, инфляция, управление, принятие решений, инвестиционный проект.
\end{abstract}

Учет воздействия инфляции является одной
из проблем оценки $\quad$ эффективности инвестиционных проектов. Решение данной задачи усложняется, когда уровень инфляции не представляется возможным оценить с достаточным уровнем определенности.

Воздействие инфляции на притоки и оттоки финансового потока проекта несомненно оказывает влияние на эффективность инвестиционного проекта и возможность его финансовой реализуемости, так как обесценивание денежной единицы, используемой в инвестиционных расчетах, вызывает необходимость в дополнительном финансировании.

Влияние инфляции, как и элементов риска при реализации инвестиционного проекта, необходимо учитывать с помощью проведения анализа чувствительности на основе метода варьирования параметров, используемых при расчете показателей эффективности проекта.

При оценке эффективности инвестиционного проекта осуществляется проверка полученных значений показателей эффективности проекта требованиям ЛПР [2 - 4]. В случае, если эти требования не выполняются, либо выбирается новый проект, либо финансовая схема текущего проекта подвергается необходимой корректировке. Если требования ЛПР выполнены, то производится анализ чувствительности проекта, т.е. с помощью компьютерной модели получают набор значений показателей эффективности данного проекта для различных вариантов возможных изменений 
параметров проекта. Экономический анализ полученных результатов позволит получить системную оценку «устойчивости» проекта по отношению к изменению внешних и внутренних условий его реализации.

В целях получения системной оценки изменений показателей эффективности проекта в различных условиях его реализации (как внутренних, так и внешних) необходимо учесть влияние такого важного внешнего фактора, как инфляция.

Из рис. 1 следует, что при анализе чувствительности проекта в условиях инфляции необходимо модифицировать экономикоматематическую модель оценки инвестиционного проекта [7, 8], чтобы учесть в них зависимость функций-критериев и функций-ограничений от уровня инфляции, который задается величиной $h$ (в \% или долях единицы за период). Оценка количественного влияния уровня инфляции на конечные результаты финансовых операций, в которых

главную роль играют потоки платежей, является столь же важной, сколь и сложной задачей одновременно. Важность этой задачи, по сути дела, определяется ее актуальностью, поскольку следствием инфляции является реальное обесценивание денежной единицы и, соответственно, падение покупательной способности, что, в свою очередь, вызывает реальное изменение показателей конечных результатов финансовых операций.

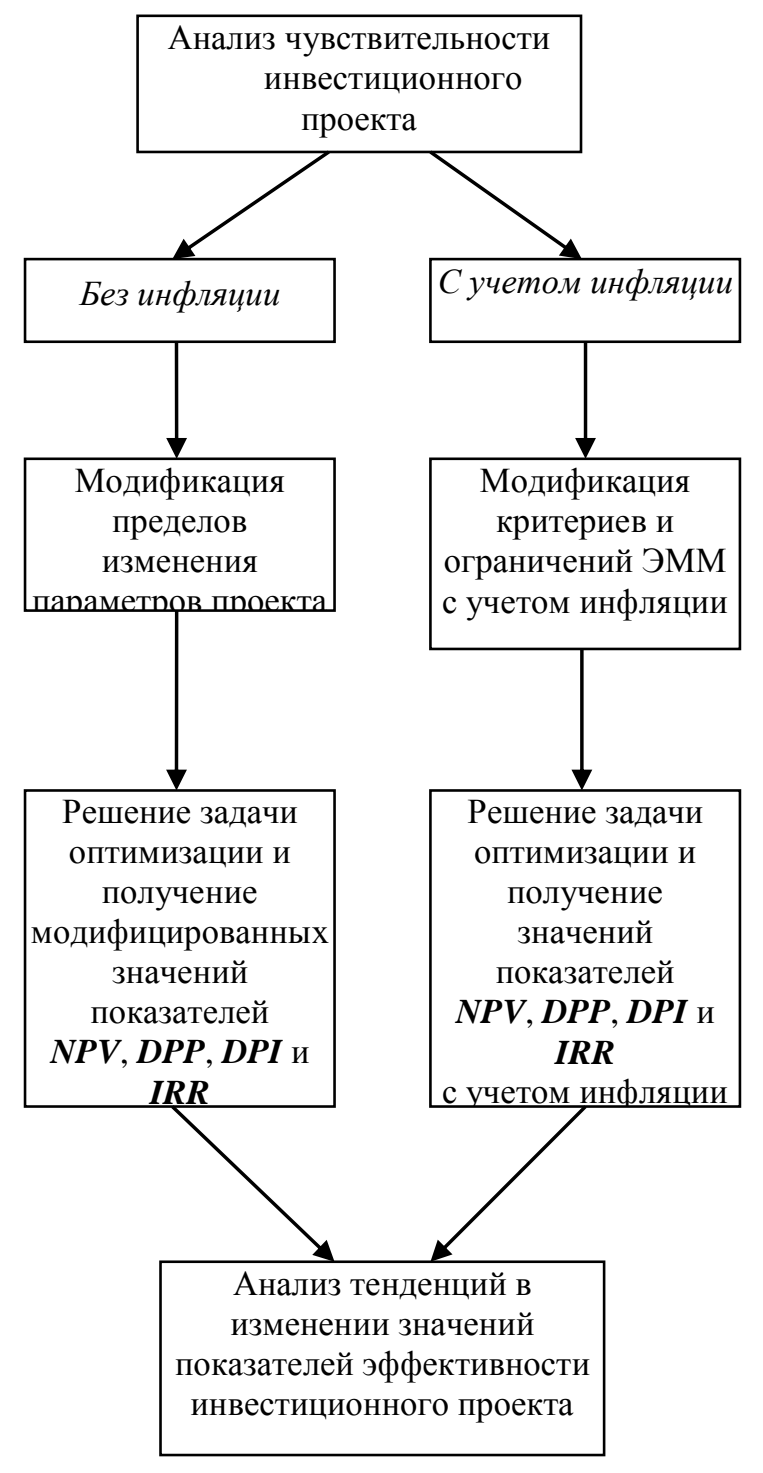

Рисунок 1 - Схема анализа чувствительности. 
Сложность этой задачи определяется далеко неоднозначным изменением конечных результатов финансовых операций, полученных с учетом уровня инфляции и без него. Экономический смысл инфляционных процессов говорит о том, что конечный результат операции станет меньше, поскольку его часть будет «съедена» инфляцией. Однако, в зависимости от выбора точки приведения для вычисления результата операции (что очень важно для задач финансовой математики), типа потоков платежей и других параметров значения конечных результатов, полученных до учета инфляции и после него могут заметно отличаться друг от друга, причем как в большую, так и в меньшую стороны. Именно в этом выражается неоднозначность изменения конечных результатов финансовых операций, связанных с использованием потоков платежей, к которым, без сомнения, относятся инвестиционные проекты $[5,6]$.

Возможно, именно такими причинами объясняется отсутствие анализа влияния инфляции на показатели эффективности инвестиционных проектов, которое имеет место в бизнес-планах реальных проектов [9, 10]. Таким образом, серьезное противоречие, состоящее в том, что учет и анализ влияния инфляции, важность которого уже никому не надо доказывать, отсутствует при оценке эффективности реальных инвестиционных проектов, вызывает необходимость рассмотреть этот вопрос более подробно.

Пусть однократный платеж $P_{0}$, сделанный в момент $t=0$, наращивается в условиях инфляции и без нее как показано на рис. 2.

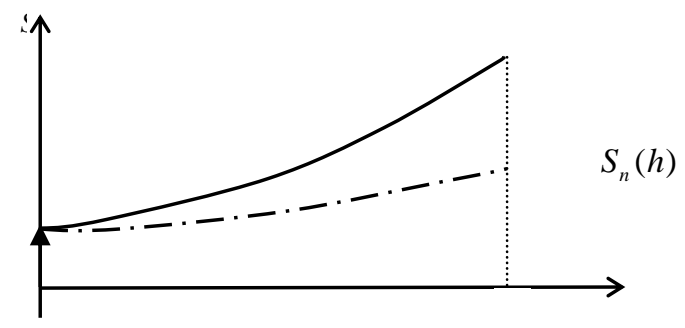

\section{Рисунок 2 - Схема наращения однократного платежа.}

Тогда, очевидно, в соответствии с правилами финансовой математики [1], величина наращенного к моменту $t=n$ платежа с учетом инфляции $S_{n}(h)$ :

$$
S_{n}(h)=P_{0} \cdot\left(\frac{1+r}{1+h}\right)^{n},
$$

где $r$ - размер нетто - ставки наращения. Но, также очевидно, что без инфляции величина наращенного платежа $S_{n}$ к моменту $t=n$ будет:

$$
S_{n}=P_{0} \cdot(1+r)^{n} \text {. }
$$

Отсюда следует, что $P_{0}=\frac{S_{n}}{(1+r)^{n}}$ и

$$
S_{n}(h)=\frac{S_{n}}{(1+r)^{n}} \cdot\left(\frac{1+r}{1+h}\right)^{n}=\frac{S_{n}}{(1+h)^{n}} .
$$

Из (1) понятно, что величина наращенного платежа в условиях инфляции уменьшается в $(1+h)^{n}$ раз, по сравнению с той же величиной без учета инфляции.

Рассмотрим теперь обратную задачу: какова должна быть величина начального платежа без инфляции $-P_{0}$, и с ее учетом $-P_{0}(h)$, чтобы наращение в обоих случаях приводило к одному и тому же результату $S_{n}$ как показано на рис. 3 .

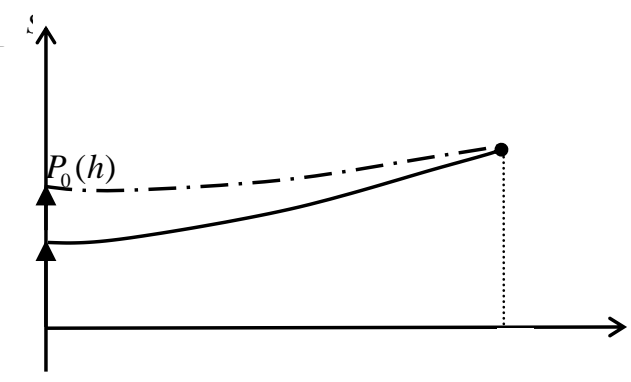

Рисунок 3 - Схема наращения начального платежа. 
Очевидно, что в этом случае справедливы соотношения:

$$
S_{n}=P_{0} \cdot(1+r)^{n}=P_{0}(h) \cdot\left(\frac{1+r}{1+h}\right)^{n},
$$

откуда следует, что

$$
P_{0}(h)=P_{0} \cdot(1+h)^{n} .
$$

Таким образом, выражение (2) показывает, что приведенная стоимость одного и того же платежа с учетом инфляции увеличивается относительно той же величины в отсутствии инфляции в $(1+h)^{n}$ раза.

Естественно, полученные выше результаты скажутся на значении показателей эффективности инвестиционного проекта в условиях инфляции, так как в основе их оценки лежит совокупность приведенных стоимостей отдельных платежей, составляющих потоки платежей проекта. Однако, поскольку эта совокупность представляет собой не сумму, а разность приведенных стоимостей платежей доходной и инвестиционной частей, влияние инфляции на конечные результаты проекта не будет столь однозначным, как показано выше в случае однократных платежей.

Рассмотрим определение показателя $N P V$ инвестиционного проекта с учетом инфляции с темпом $h$ (в долях единицы) в год. Пусть финансовая схема реализации проекта будет такой, как показано на рис. 4 , где $P_{t}\left(t=1, \ldots, n_{1}\right)-$ приведенные стоимости платежей инвестиционной части без учета инфляции, а $P_{t}$ ( $\left.t=n_{1}+1, \ldots, n_{1}+n_{2}\right) \quad$ - приведенные стоимости платежей доходной части без учета инфляции.

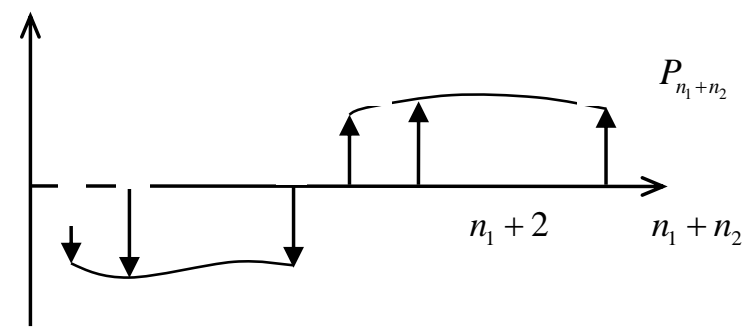

Рисунок 4 - Условная финансовая схема реализации проекта.

Тогда, используя здесь (2), можно записать выражение для приведенного чистого дохода проекта с учетом инфляции в форме:

$$
\begin{aligned}
N P V(h) & =\left[\begin{array}{l}
P_{n_{1}+1} \cdot(1+h)^{n_{1}+1}+P_{n_{1}+2} \cdot(1+h)^{n_{1}+2}+ \\
+\ldots+P_{n_{1}+n_{2}} \cdot(1+h)^{n_{1}+n_{2}}
\end{array}\right]- \\
& -\left[P_{1} \cdot(1+h)+P_{2} \cdot(1+h)^{2}+\ldots+P_{n_{1}} \cdot(1+h)^{n_{1}}\right],
\end{aligned}
$$

а для приведенного чистого дохода без учета инфляции в форме:

$$
N P V=\left[P_{n_{1}+1}+P_{n_{1}+2}+\ldots+P_{n_{1}+n_{2}}\right]-\left[P_{1}+P_{2}+\ldots+P_{n_{1}}\right] .
$$

Для того чтобы сравнить величины $N P V(h)$ и $N P V$ оценим величину разности между ними:

$$
\begin{aligned}
\triangle N P V(h) & =N P V(h)-N P V=\left\{\begin{array}{l}
P_{n_{1}+1} \cdot\left[(1+h)^{n_{1}+1}-1\right]+ \\
+P_{n_{1}+2} \cdot\left[(1+h)^{n_{1}+2}-1\right]+\ldots+
\end{array}\right. \\
& \left.+P_{n_{1}+n_{2}} \cdot\left[(1+h)^{n_{1}+n_{2}}-1\right]\right\}- \\
-\left\{P_{1} \cdot[(1+h)-1]+P_{2} \cdot\left[(1+h)^{2}-1\right]+\ldots+\right. & \\
& \left.+P_{n_{1}} \cdot\left[(1+h)^{n_{1}}-1\right]\right\} .
\end{aligned}
$$

Очевидно, что знак величины $\triangle N P V(h)$ будет зависеть от значений параметров $n_{1}$ и $n_{2}$, а также от соотношения размеров платежей инвестиционной и доходной частей, то есть, в общем случае, от финансовой схемы реализации конкретного инвестиционного проекта.

Рассмотрим случай, когда $n_{1}=n_{2}$, тогда выражение для оценки величины $\triangle N P V(h)$ можно записать в следующей форме:

$$
\begin{aligned}
& \triangle N P V(h)=\left\{\begin{array}{l}
P_{n_{1}+1} \cdot\left[(1+h)^{n_{1}+1}-1\right]- \\
-P_{1} \cdot[(1+h)-1]
\end{array}\right\}+ \\
&+\left\{\begin{array}{l}
P_{n_{1}+2} \cdot\left[(1+h)^{n_{1}+2}-1\right]- \\
-P_{2} \cdot\left[(1+h)^{2}-1\right]
\end{array}\right\}+\ldots \\
& \ldots+\left\{P_{n_{1}+n_{1}} \cdot\left[(1+h)^{n_{1}+n_{1}}-1\right]-P_{n_{1}} \cdot\left[(1+h)^{n_{1}}-1\right]\right\}
\end{aligned}
$$

что соответствует сумме

$$
\triangle N P V(h)=\sum_{k=1}^{n_{1}}\left\{\begin{array}{l}
P_{n_{1}+k} \cdot\left[(1+h)^{n_{1}+k}-1\right]- \\
-P_{k} \cdot\left[(1+h)^{k}-1\right]
\end{array}\right\},
$$


Из анализа (4) следует, что величина $\triangle N P V(h)$ будет положительной в случае, если выполняется условие

$$
P_{n_{1}+k} \cdot\left[(1+h)^{n_{1}+k}-1\right]-P_{k} \cdot\left[(1+h)^{k}-1\right]>0,
$$

а это достигается в случае, если приведенные стоимости платежей инвестиционной и доходной частей проекта удовлетворяют соотношению

$$
\frac{P_{n_{1}+k}}{P_{k}}>\frac{(1+h)^{k}-1}{(1+h)^{n_{1}+k}-1}, k=1,2, \ldots, n_{1} .
$$

Для того, чтобы получить аналогичное соотношение между номинальными размерами платежей инвестиционной $\left(x_{k}\right)$ и доходной $\left(y_{n_{1}+k}\right.$ ) частей проекта, необходимо определить их приведенные стоимости, используя нетто-ставку дисконтирования $r$ :

$$
P_{n_{1}+k}=\frac{y_{n_{1}+k}}{(1+r)^{n_{1}+k}}, P_{k}=\frac{x_{k}}{(1+r)^{k}} .
$$

Используя последние выражения в (6), получаем соотношение между номинальными размерами платежей инвестиционной и доходной частей проекта

$$
\frac{y_{n_{1}+k}}{x_{k}}>\frac{(1+h)^{k}-1}{(1+h)^{n_{1}+k}-1} \cdot(1+r)^{n_{1}},
$$

при котором $\triangle N P V(h)>0$. Таким образом, если длительности инвестиционной и доходной частей проекта совпадают, а размеры платежей этих частей удовлетворяют условию (7), величина показателя приведенного чистого дохода проекта с учетом инфляции будет больие этого показателя в условиях отсутствия инфляции при прочих равных условиях.

Рассмотрим случай, когда $n_{1}>n_{2}$, причем $n_{1}=n_{2}+n_{3}$. Выполняя вычисления $N P V(h)$ и $N P V$ аналогично тому, как это было сделано выше, выражение для оценки величины $\triangle N P V(h)$ в этом случае можно записать в форме, аналогичной выражению (4):

$$
\begin{aligned}
\triangle N P V(h) & =\sum_{k=1}^{n_{2}}\left\{\begin{array}{l}
P_{n_{1}+k} \cdot\left[(1+h)^{n_{1}+k}-1\right]- \\
-P_{k} \cdot\left[(1+h)^{k}-1\right]
\end{array}\right\}- \\
& -\sum_{k=1}^{n_{3}}\left\{P_{n_{2}+k} \cdot\left[(1+h)^{n_{2}+k}-1\right]\right\} .
\end{aligned}
$$

Из анализа (8) следует, что величина $\triangle N P V(h)$ будет положительной в случае, если будут одновременно выполняться 2 условия:

$$
\begin{gathered}
P_{n_{1}+k} \cdot\left[(1+h)^{n_{1}+k}-1\right]-P_{k} \cdot\left[(1+h)^{k}-1\right]>0, \\
k=1,2, \ldots, n_{2} ;
\end{gathered}
$$

2)

$$
\begin{aligned}
& \sum_{k=1}^{n_{2}}\left\{\begin{array}{l}
P_{n_{1}+k} \cdot\left[(1+h)^{n_{1}+k}-1\right]- \\
-P_{k} \cdot\left[(1+h)^{k}-1\right]
\end{array}\right\}> \\
& >\sum_{k=1}^{n_{3}}\left\{P_{n_{2}+k} \cdot\left[(1+h)^{n_{2}+k}-1\right]\right\}
\end{aligned}
$$

Очевидно, что и в этом случае можно получить соотношения между номинальными размерами платежей обеих частей проекта, аналогичные выражению, для того, чтобы $\triangle N P V(h)$ была положительной величиной, однако аналитические выражения будут гораздо сложнее и здесь не приводятся. Таким образом, если длительность инвестиционной части больше длительности доходной части проекта, а размеры приведенных стоимостей платежей обеих частей удовлетворяют условиям (9) - (10), величина показателя приведенного чистого дохода проекта с учетом инфляции также будет больше этого показателя в условиях отсутствия инфляции при прочих равных условиях.

Рассмотрим случай, когда $n_{2}>n_{1}$, причем $n_{2}=n_{1}+n_{3}$. Выполняя вычисления $N P V(h)$ и $N P V$ аналогично тому, как это было сделано выше, выражение для оценки величины $\triangle N P V(h)$ в этом случае можно записать в форме, аналогичной выражению (8):

$$
\begin{aligned}
\triangle N P V(h) & =\sum_{k=1}^{n_{1}}\left\{P_{n_{1}+k} \cdot\left[(1+h)^{n_{1}+k}-1\right]-P_{k} \cdot\left[(1+h)^{k}-1\right]\right\}+ \\
& +\sum_{k=1}^{n_{3}}\left\{P_{n_{1}+k} \cdot\left[(1+h)^{n_{1}+k}-1\right]\right\} .
\end{aligned}
$$

Из анализа (11) следует, что величина $\triangle N P V(h)$ будет положительной в случае, если выполняется условие аналогичное (5)

$$
P_{n_{1}+k} \cdot\left[(1+h)^{n_{1}+k}-1\right]-P_{k} \cdot\left[(1+h)^{k}-1\right]>0,
$$

а это достигается в случае, если приведенные стоимости платежей инвестиционной и доходной частей проекта удовлетворяют соотношению (6), a для номинальных значений этих платежей соответственно выполняется условие (7).

Естественно, что если условия положительности $\triangle N P V(h)$, полученные в каждом из рассмотренных выше случаев, не выполняются, то становится очевидным, что значение показателя приведенного чистого дохода с учетом инфляции будет меньше значения этого же показателя в условиях отсутствия инфляции. По нашему мнению именно этой неоднозначностью «поведения» показателя $N P V(h)$ в отличие от показателя $N P V$ объясняется отсутствие анализа чувствительности с учетом инфляции в бизнеспланах реальных инвестиционных проектов.

Однако, несмотря на математические сложности, приведенные при оценке показателя 
$\triangle N P V(h), \quad$ экономическая суть такого «поведения», на наш взгляд, вполне прозрачна. Экономический смысл потока платежей инвестиционного проекта, в конечном счете, состоит в извлечении прибыли из этой финансовой операции с желаемой нормой $r$. Тогда приведенная стоимость любого из платежей потока, сделанного в момент $t=n, \mathrm{c}$ экономической точки зрения представляет такой размер вклада на депозит по ставке $r$, сделанный в момент $t=0$, который к моменту $t=n$ вырастет ровно в $(1+r)^{n}$ раз. Но поскольку часть вклада за время наращения «съест» инфляция, для получения желаемой прибыли необходимо увеличить его начальный размер. Собственно, такой же экономический принцип положен в основу известной в инвестиционном анализе формулы Фишера [1], которая определяет размер надбавки к нетто-ставке $r$ для достижения необходимого результата в условиях инфляции с темпом $h$.
Для схемы инвестирования рис. 4.1 в соответствии с правилами финансовой математики [1] показатель $N P V$ будет учитывать влияние инфляции, если

$$
\begin{aligned}
N P V\left(x_{t}, y_{t}, r, h\right)= & P\left(y_{t}, r, h\right)-P\left(x_{t}, r, h\right),(12 \\
\text { где } \quad P\left(y_{t}, r, h\right) & =\sum_{t=n_{1}+1}^{n_{1}+n_{2}} \frac{y_{t}}{(1+r)^{t}} \cdot(1+h)^{t}
\end{aligned}
$$

приведенная к моменту $t=0$ стоимость доходных платежей проекта с учетом инфляции, а $P\left(x_{t}, r, h\right)=\sum_{t=1}^{n_{1}} \frac{x_{t}}{(1+r)^{t}} \cdot(1+h)^{t}-$ приведенная к моменту $t=0$ стоимость инвестиционных платежей проекта с учетом инфляции.

При постоптимизационном анализе чувствительности проекта темп инфляции $\mathbf{h}$ будет использоваться, наряду с другими параметрами проекта, в качестве управляющей переменной для получения ряда значений показателей эффективности проекта в различных внешних условиях роста цен.

\section{References:}

1. Chetyrkin EM (2011) Finansovaya matematika. - Moscow: ID «Delo» RANKhiGS, 2011. $392 \mathrm{p}$.

2. Dosuzheva EE, Kirillov YV (2014) K voprosu o ratsional'nom podkhode $\mathrm{k}$ prinyatiyu upravlencheskikh resheniy. - Idei i idealy. 2014. - № 1 (19), t. 2. - pp. 89-98.

3. Dosuzheva EE, Kirillov YV (2014) Osnovnye printsipy realizatsii investitsionnogo proekta. Naukovedenie: internet-zhurnal. - 2014. - № 2. Available:

http://naukovedenie.ru/PDF/138EVN214.pdf (Accessed: 20.04.2015).

4. Dosuzheva EE, Kirillov YV (2014) Sistemnyy podkhod $\mathrm{k}$ razrabotke upravlencheskikh resheniy pri investirovanii. - Naukovedenie: internet-zhurnal. - 2014. - №1. Available: http://naukovedenie.ru/PDF/04EVN114.pdf (Accessed: 20.04.2015).

5. Frolova TA (2005) Ekonomika predpriyatiya: konspekt lektsiy. - Taganrog: Izd-vo TRTU, 2005. -320 p.
6. Khazanovich ES (2009) Inostrannye investitsii. - Moscow: Knorus, 2009. - 320 p.

7. Kirillov YV, Dosuzheva EE (2013) Ekonomiko-matematicheskaya model' podderzhki prinyatiya resheniy po investirovaniyu $\mathrm{v}$ sovmestnye investitsionnye proekty. - Finansovaya analitika: problemy i resheniya. - 2013. - № 27. - pp. 33-39.

8. Kirillov YV, Dosuzheva EE (2013) Mnogokriterial'naya ekonomikomatematicheskaya model' otsenki kommercheskoy effektivnosti investirovaniya. Finansovaya analitika: problemy i resheniya. 2013. - № 32. - pp. 18-24.

9. (2014) Kollektsiya biznes-planov real'nykh proektov. $\quad$ - $\quad$ Available: http://www.cfin.ru/business-plan/samples/ (Accessed: 01.10.2014).

10. (2014) Ofitsial'nyy sayt kompanii «Shtoller Konsalting». Primery biznes-planov proektov. Available: http://www.teo.ru/project.htm/ (Accessed: 01.10.2014) 